\title{
Revisão: Comportamento Suicida ao Longo do Ciclo Vital
}

\author{
Adriano Schlösser ${ }^{1}$ \\ Gabriel Fernandes Camargo Rosa \\ Programa de Pós-Graduação em Psicologia da Universidade Federal de Santa Catarina, \\ Florianópolis, Santa Catarina, Brasil \\ Carmen Leontina Ojeda Ocampo More \\ Departamento de Psicologia da Universidade Federal de Santa Catarina, Florianópolis, \\ Santa Catarina, Brasil
}

\begin{abstract}
Resumo
O comportamento suicida pode ser considerado como sendo o ato intencional de causar dano a si mesmo, sendo o objetivo final dar cabo a própria vida, englobando idéias e desejos suicidas, condutas suicidas sem resultado de morte e os suicídios consumados. O presente artigo objetiva revisar estudos sobre o comportamento suicida ao longo do ciclo vital, identificando os possíveis fatores de risco e proteção característicos de cada etapa. Foram selecionadas e analisadas 66 referências, com publicações entre os anos de 2000 a 2012, abarcando três categorias do ciclo vital: fase infanto-juvenil, fase adulta e fase idosa. Como resultados, observaram-se generalidades quanto a fatores de risco e protetivos, uma vez que estão presentes em todas as etapas do desenvolvimento humano, bem como fatores específicos, que se apresenta particularmente em cada fase do ciclo vital.
\end{abstract}

Palavras-chave: Comportamento suicida, suicídio, ciclo vital.

\section{Review: Behavior Suicidal Throughout the Life Sycle}

\begin{abstract}
Suicidal behavior can be regarded as the act of intentionally causing harm to oneself, the ultimate goal being to end one's own life. It includes suicidal ideas and desires, suicidal behaviors not resulting death and actual suicides. The present article reviews sixty six studies on suicidal behavior throughout the life cycle identifying possible risk factors and protective characteristics of each stage. The publications were from the years 2000 to 2012, they were selected and analyzed, including the three categories of the life cycle: the infant to teenager stage, adulthood and elder phase. General risk and protective factors were observed since they are present in all stages of human development, as well as specific factors, which are particularly present in each phase of the life cycle.
\end{abstract}

Keywords: Suicidal behavior, suicide, life cycle.

\section{Revision: Comportamiento Suicida Durante el Ciclo de Vida}

\section{Resumen}

El comportamiento suicida puede ser considerado como el acto intencional de causar daño a sí mismo, con objetivo último de dar a quitar su vida, abarcando las ideas y deseos suicidas, conductas suicidas sin

Endereço para correspondência: Rua José Antônio Salles, 305, Jardim Iririú, Joinville, SC, Brasil 89224-300. E-mail: adriano.psicologia@yahoo.com.br, gabrielfcrosa@hotmail.com e carmenloom@gmail.com 
resultado de muerte y suicidio. En el presente artículo se revisan los estudios sobre el comportamiento suicida durante todo el ciclo de vida, la identificación de posibles factores de riesgo y características de protección de cada etapa. Se seleccionaron y analizaron 66 referencias, publicaciones entre los años 2000 y 2012, abarcando tres categorías del ciclo de vida: fase de la etapa juvenil, la edad adulta y la vejez. Como resultados, se observa a factores de riesgo generales y de protección ya que están presentes en todas las etapas del desarrollo humano, así como de factores específicos, que son particularmente presente en cada fase del ciclo de vida.

Palabras clave: Comportamiento suicida, suicidio, ciclo vital.

O suicídio é apresentado como grave problema de saúde pública, estando entre as dez principais causas de morte na população mundial de todas as faixas etárias (V. dos S. Souza et al., 2011). Além disso, o suicídio vem ocupando o terceiro lugar no grupo com idade entre 15 e 34 anos e o segundo lugar em pessoas acima de 65 anos, com taxas variando em função do contexto social, gênero, meios utilizados e faixa etária (Baggio, Palazzo, \& Aerts, 2009; Evans, Hawton, \& Deeks, 2005; Rábago, Flores, Ureña, Vargas, \& Gámez, 2010; L. D. de M. Souza et al., 2010; Wagner, Ireland \& Resnik, 2001). Nos últimos anos, tal fenômeno tem sofrido incremento significativo, principalmente nas populações juvenis, mas em grande medida passível de prevenção (Macente \& Zandonade, 2010; V. dos S. Souza et al., 2011).

Segundo a Organização Mundial de Saúde - OMS (World Health Organization [WHO], 2001), quase metade de todas as mortes violentas são em decorrência do suicídio, traduzindo-se em quase 1 milhão de vítimas ao ano, e entre 10 a 20 milhões de pessoas tentam suicídio por ano. As estimativas realizadas apontam que, em 2020 , as vítimas poderiam ascender a 1,53 milhões, e de 10 a 20 vezes mais pessoas realizarão intento suicida (Baptista, 2004; Psic, 2011; L. D. de M. Souza et al., 2010).

Estudo realizado por Wasserman, Cheng e Jiang (2008) sobre taxas de suicídio entre jovens, com dados de 90 países, estimou em 7,4/100 mil a taxa de suicídios entre jovens de 15 e 19 anos. Entre aqueles com maiores taxas estão: Sri Lanka (46,5/100 mil), Lituânia $(23,9 / 100$ mil) e Rússia (23,6/100 mil). No Brasil, a estimativa foi de 4,2/100 mil, considerada baixa em escala mundial, ocupando assim o $71^{\circ}$ lugar. Foram constatadas também maiores taxas entre jovens do sexo masculino (10,5/100 mil) do que do sexo feminino (4,1/100 mil), com a Região Sul apresentando médias acima das demais regiões do país, encabeçadas por RS, com 11/100 mil (Baggio et al., 2009; Werlang \& Botega, 2004). Contudo, vale ressaltar que tais dados precisam ser cuidadosamente pontuados, uma vez que o suicídio, por ser um tema tabu para a sociedade brasileira, passa a ser subnotificado, gerando assim informações imprecisas quanto aos casos.

Num estudo realizado com a população brasileira entre 1980 e 2000, verificou-se um aumento de $32,8 \%$ na taxa masculina de suicídio, com crescimento em todos os grupos etários; Entre as mulheres, as taxas são mais altas em planejamento e tentativa de suicídio, enquanto no sexo masculino as maiores taxas são de suicídio consumado. (Baggio et al., 2009).

Operacionalmente, o suicídio pode ser definido como sendo "dar fim na própria vida voluntariamente", consistindo em um fenômeno paradoxal que desafia várias áreas do conhecimento, tais como: Psicologia, Filosofia, Direito, Psiquiatria, Bioética, bem como diversas religiões e o senso comum (Daolio \& Silva, 2009). Ademais, o fenômeno do suicídio é multicausal, envolvendo fatores ambientais, psicológicos, culturais, biológicos e políticos, tudo englobado na existência do indivíduo.

É importante assinalar que o suicídio se apresenta como consequência final de um leque maior de situações que põem em risco a vida, considerando-as como comportamentos suicidas. O comportamento suicida pode ser definido como a preocupação, desejo ou ato que busca, intencionalmente, causar dano a si mesmo. 
Abarcam neste quesito as ideias e desejos suicidas (ideação suicida), os comportamentos (ou condutas) suicidas sem resultado de morte e/ou os suicídios consumados (Psic, 2011; Werlang, Borges, \& Fenterseifer, 2005).

Dadas essas considerações, algumas questões se fazem presente: Como costumam ocorrer os comportamentos suicidas? Suas formas de manifestação são sempre as mesmas, independentemente da idade?

É neste momento que se passa a refletir o ser humano sob uma perspectiva atrelada ao ciclo vital que, por sua vez, é definido como um conjunto de fases pelas quais o indivíduo atravessa, desde a sua concepção até o seu desaparecimento, sendo que, em cada uma delas, peculiaridades são passíveis de observação. Cada ser humano traz consigo características específicas, potencialidades, dificuldades e demais constructos biopsicossociais que configuram cada etapa da vida, necessitando de um olhar que explore melhor cada um destes estágios (Alarcão, 2006).

Com efeito, o objetivo desta pesquisa visa revisar estudos acerca do comportamento suicida ao longo do ciclo vital, atravessando suas três fases: fase infanto-juvenil, adulta e fase idosa. Deste modo, a proposta é a de entender se há e quais seriam as especificidades de cada etapa em relação ao comportamento suicida, com os fatores potencialmente de risco e protetivos característicos de cada período.

\section{Método}

Tendo em vista que o objetivo do presente trabalho é fazer uma revisão de literatura sobre o comportamento suicida ao longo do ciclo vital, foi realizada uma revisão sistemática de pesquisas referentes ao tema. A busca textual sobre comportamento suicida foi coletada a partir das bases de dados brasileira Biblioteca Virtual em Saúde (BVS-Psi), bem como da base de dados internacional PsycINFO (base de dados da American Psychological Association), abrangendo publicações a partir do ano 2000 até 2012.

Os descritores utilizados para a busca e recuperação de artigos foram: "comportamento suicida" e "suicídio" nas bases nacionais e "suicidal behavior" e "suicide" nas bases internacionais. Selecionaram-se estudos que adentravam na temática do comportamento suicida nos mais diferentes enfoques: epidemiológico, fatores associados ao comportamento suicida, programas de prevenção, fatores potencialmente protetivos e de risco.

Para tanto, pesquisas quantitativas, qualitativas, revisões de literatura e estudos clínicos foram introduzidos na seleção do material. Para critérios de seleção, buscou-se incluir estudos publicados em português, inglês e espanhol, dentro do período já citado, cuja temática estivesse voltada a algum dos descritores utilizados.

Foram contabilizados ao todo 901 artigos, sendo 149 deles publicações nacionais. De posse dos dados, calcularam-se as frequências totais dos artigos por ano, incluindo os artigos internacionais. Posteriormente, optou-se pela realização da leitura dos resumos dos artigos, objetivando assim a seleção apenas das publicações que atendessem às propostas deste estudo.

Vencida a etapa de recuperação, seleção e leitura, foi realizado o fichamento para condensar os dados obtidos. A partir disto, a análise e interpretação do material foi dividida em quatro fases, conforme ponderam Lakatos e Marconi (2003): (a) apreciação crítica do material; (b) decomposição dos elementos essenciais; (c) agrupamento e classificação; (d) análise final.

Os dados foram analisados de modo qualitativo, sendo apresentados, ao longo do estudo, os principais aspectos encontrados sobre a temática "comportamento suicida" no ciclo vital. Nas discussões, optou-se por apresentar, primeiramente, uma comparação entre a produção nacional e a internacional de artigos, de acordo com a frequência de publicação entre 2000 e 2012. Em seguida, através da seleção de publicações, discutiu-se o comportamento suicida ao longo do ciclo vital em três eixos: "População infanto-juvenil"; "População adulta" e "População Idosa".

\section{Resultados e Discussão}

Os resultados estão organizados em dois tópicos. No primeiro é realizado um comparativo 
entre a produção nacional e internacional entre 2000 e 2012, mediante a verificação da frequência de publicações desta temática. Posteriormente, é feita a discussão acerca do comportamento suicida ao longo do ciclo vital nas suas diferentes etapas.

\section{Produção Internacional e Nacional}

De modo geral, foram contabilizados 752 artigos internacionais, contendo a discussão sobre o suicídio no período entre 2000 e 2012. Neste mesmo período, conforme se observa na Figura 1, a produção nacional realizou 149 publicações desta temática, abarcando $19,8 \%$ do total de publicações verificadas.

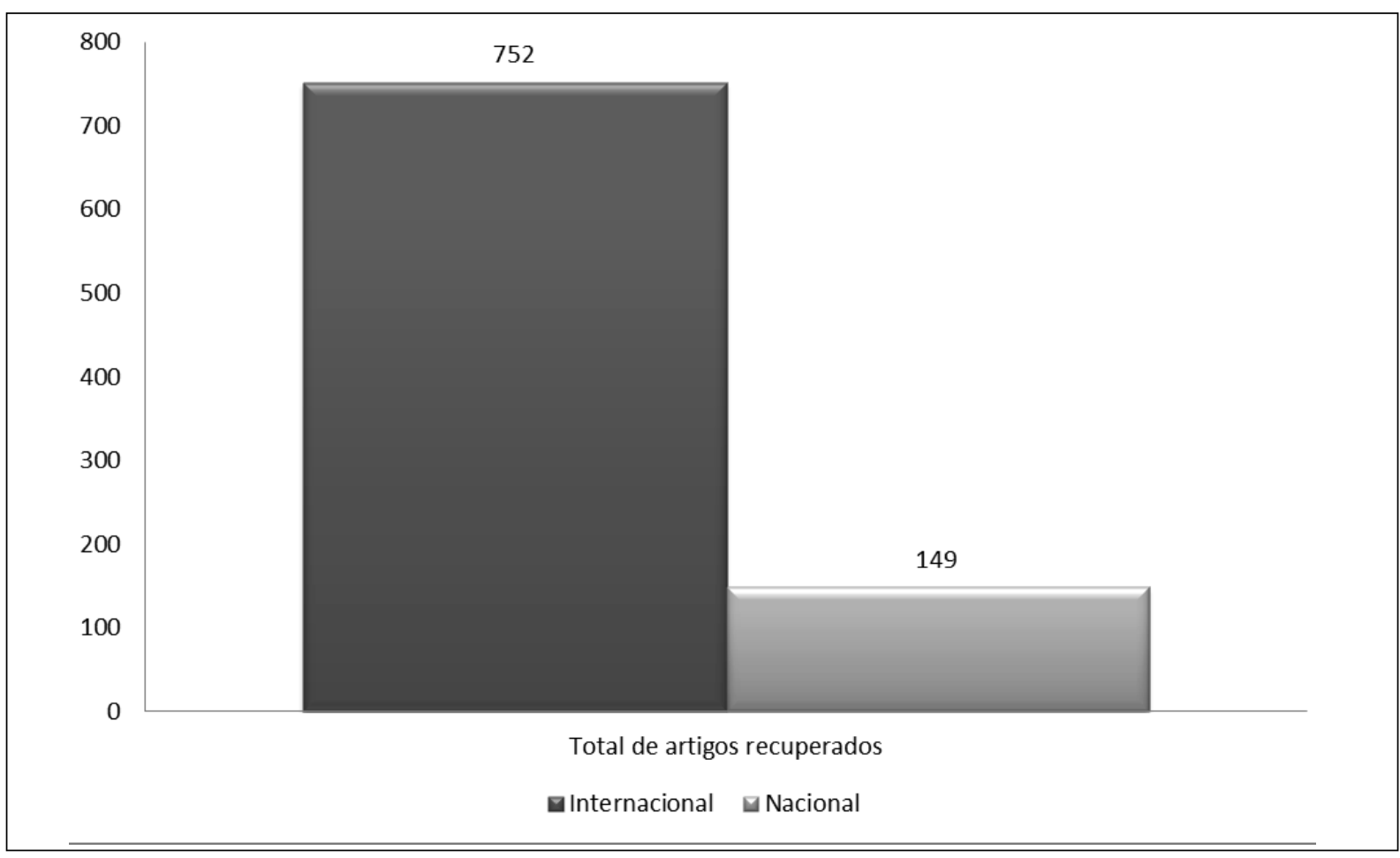

Figura 1. Totalidade dos artigos recuperados, separados em nacionais e internacionais.

A partir da base de dados internacional BVS-Psi, verificou-se que a produção desta temática vem se mantendo relativamente estável ao longo da década, com maior pico produtivo em 2008, mas conservando a quantidade de publicações de forma equilibrada. Por sua vez, a base de dados internacional PsycINFO apresentou uma baixa frequência de publicações até 2004. A partir de 2005, houve um discreto aumento no número de trabalhos, com 7 publicações no referido ano e chegando a 8 , no ano de 2007, com queda em 2009, mantendo a estabilidade nos anos seguintes.

Por sua vez, a publicação de periódicos nacionais sobre suicídio merece destaque. Até o ano de 2003, a produção nacional apresentou baixa frequência de publicações, com até 3 pu- blicações ao ano. A partir de 2004, observou-se crescente aumento nas publicações, chegando ao pico, em 2010, com 35 publicações neste período. Assinala-se, aqui, que este crescimento, mesmo considerável, não ocorreu de forma linear, uma vez que apresenta pequenos aumentos e quedas ao longo dos anos.

Com efeito, para a revisão de literatura, 50 artigos foram selecionados para uma análise integral. Na Tabela 1, é possível observar todas as citações - dos artigos selecionados - agrupadas de acordo com o tópico em que ele oferece contribuições. 


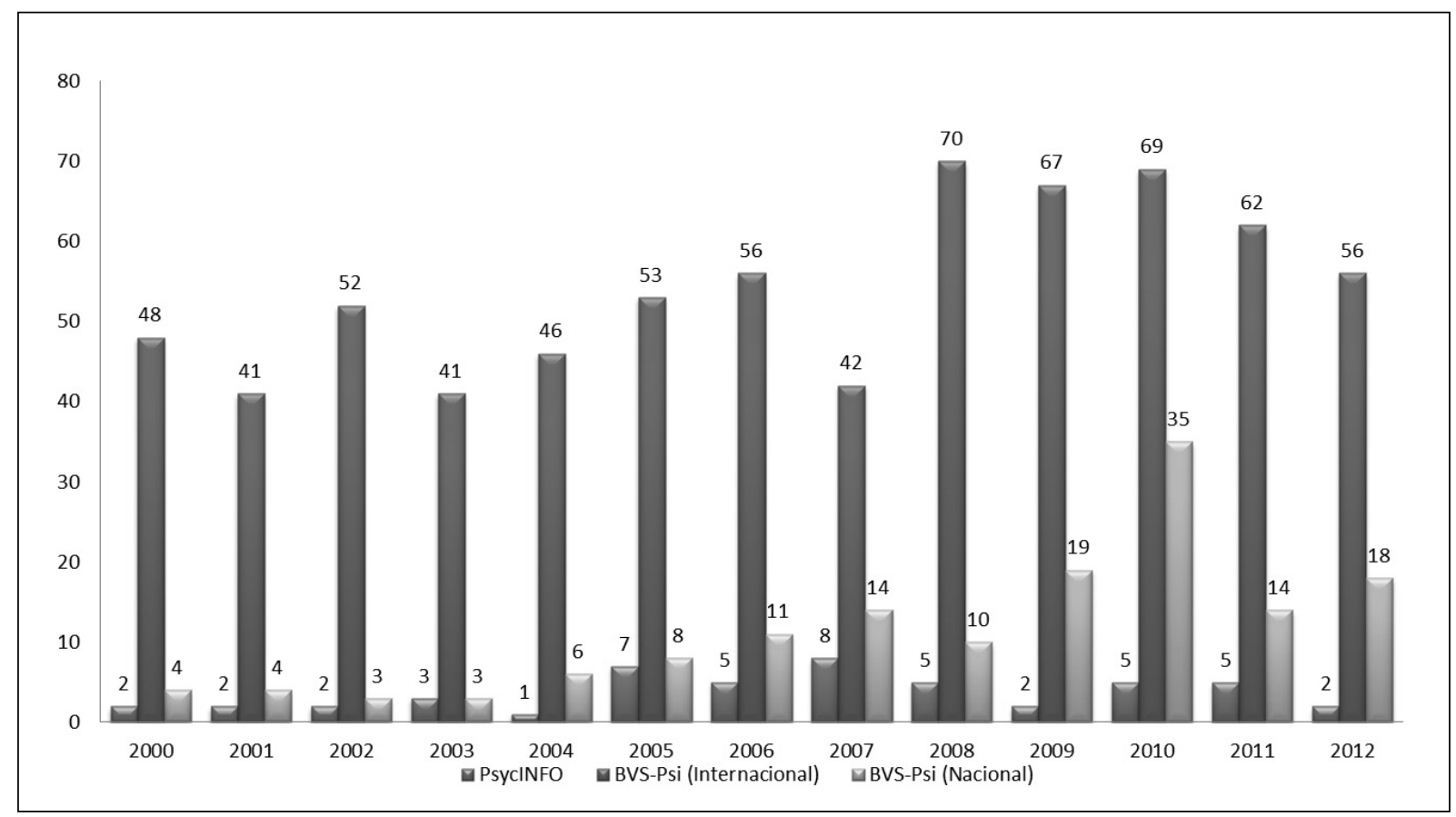

Figura 2. Distribuição dos artigos recuperados, de acordo com o ano da publicação.

\section{Tabela 1}

\section{Artigos Selecionados por Etapas do Ciclo Vital}

Etapa do Ciclo Vital Autores (Ano)

População Infanto/Juvenil Bastos (2009); Bella, Fernández, Acevedo, \& Willington (2007); Bella, (21 artigos)

Fernández, \& Willington (2010); Botega, Barros, Oliveira, Dalgalarrondo, \& Marín-León (2005); Campo et al. (2003); Carmona et al. (2010); Gómez et al. (2002); Gutiérrez, Fernández, Jiménez, Gutiérrez, \& Tamayo (2009); Joe, Romes, \& Jamieson (2007); Larranguibel, González, Martínez, \& Valenzela (2000); Mitchell \& Kim (2004); Pavez, Santander, Carranza, \& Vera-Villarroel (2009); Pelkonen \& Marttunen (2003); Pérez-Olmos, Sandoval, DussánBuitrago, \& Ayala-Aguilera (2007); Pfeffer \& Shaffer (2001); Psic (2011); Reyes \& Torres (2001); Roy (2005); Silva \& Maia (2010); Werlang et al. (2005).

População Adulta (17 artigos)

População Idosa (12 artigos)
Almeida, Guedes, Nogueira, França, \& Silva (2009); Alves, Kessler, \& Ratto (2004); Botega et al. (2009); Castro-Rueda, Rangel Martínez-Villalba, Camacho, \& Rueda-Jaimes (2010); Caycedo et al. (2010); Fanger et al. (2010); Jiang, Perry, \& Hesser (2010); King, Nardi, \& Cruz (2006); Lima et al. (2010); Marín-León \& Barros (2003); Minayo, Pinto, Assis, Cavalcante, \& Mangas (2012); Meneghel, Victora, Faria, Carvalho, \& Falk (2004); Ores et al. (2012); Rogers (2001); Sher (2010); Turecki (2001); Viana, Zenkner, Sakae, \& Escobar (2008); Weir (2001).

Baptista, Morais, Rodrigues, \& Silva (2006); Beautrais (2002); Beeston (2006); Conwell \& Thompson (2008); De Leo et al. (2001); Lovisi, Santos, Legay, Abelha, \& Valencia (2009); Minayo \& Cavalcante (2010); Minayo, Meneghel, \& Cavalcante (2012); Minayo, Pinto, et al. (2012). 


\section{População Infanto-Juvenil}

A infância e adolescência como partes do ciclo da vida humana se caracterizam por serem períodos de baixa morbidade e mortalidade. Contudo, as principais causas de morte nestas etapas estão relacionadas à: doenças oncológicas e mortes por motivos de violência, tais como homicídio e suicídio (Bella et al., 2010).

O suicídio é a terceira causa de morte entre os jovens estadunidenses (Joe et al., 2007). Na América Latina, há maior incidência de conduta suicida nos jovens entre 15 e 19 anos de idade (Gutiérrez et al., 2009), havendo estimativas que apontam para o fato de que, a cada oito jovens, um tenta se suicidar (Psic, 2011). É válido ressaltar ainda que, conforme aponta a OMS (WHO, 2001), entre os adolescentes, os comportamentos de risco ao interagir com fatores sócio-ambientais, tais como família e demais relações interpessoais, também aumentam os níveis de comportamentos suicidas.

Em outros estudos (Carmona et al., 2010; Pérez-Olmos et al., 2007), resultados denotam a adolescência como a etapa com maior índice de comportamento suicida, com médias etárias variando entre 12 e 15 anos, uma vez que se considera a adolescência como fase em que o indivíduo vivencia crises profundas, mediante as transformações, tanto físicas quanto psicológicas e culturais. Estimativas sugerem que, entre $2 \%$ e $12 \%$ da população jovem já apresentou comportamentos suicidas, o que merece considerável atenção em termos mundiais (Pelkonen \& Marttunen, 2003).

Em relação ao suicídio na infância é bem pouco relatado, com apenas quatro publicações encontradas sobre o assunto, sempre associadas à adolescência (Bella et al., 2007; Larranguibel et al., 2000; Pfeffer \& Shaffer, 2001; Psic, 2011), uma vez que a idade em que tal fenômeno passa a ocorrer é apontada a partir dos 12 anos. Sobre a intenção de morrer, na etapa da infância este dado merece consideração, haja vista que a impressão da irreversibilidade da morte não está presente antes dos oito anos de idade, já que o indivíduo está em amadurecimento neurológico e cognitivo (Bella et al., 2010).
A principal correlação para o suicídio na adolescência se apresenta mediante ideação ou intento suicida prévio, associando-se a fatores de risco, tais como a depressão maior, devido, em grande parte, a dificuldades no enfrentamento de frustrações pessoais, disfunção familiar, abuso sexual, maus tratos, bem como a não satisfação das necessidades básicas (Psic, 2011). Ademais, uso de substâncias psicoativas, perda de alguém próximo por suicídio, acesso a armas de fogo, ser vítima de algum tipo de violência, transtornos psiquiátricos, questões sócio-econômicas, problemas de interação social, abuso físico e/ou sexual, eventos estressantes, mudanças constantes de domicílio, falta de suporte social, conflitos intrafamiliares, transtornos alimentares e alterações neuropsicológicas também são apresentados como fatores potencialmente de risco na infância e adolescência (Botega et al., 2005; Gómez et al., 2002; Larranguibel et al., 2000; Pavez et al., 2009; Silva \& Maia, 2010).

Em relação ao abuso sexual infantil, seus efeitos podem manifestar-se imediatamente ou em longo prazo, podendo ocasionar, desde mudanças na estrutura e funcionamento cerebral (Roy, 2005) até transtornos psiquiátricos, tais como: transtorno de estresse pós-traumático, depressão, ideação ou intento suicida, insensibilidade emocional, disfunções sexuais, bem como problemas com substâncias psicoativas (Pérez-Olmos et al., 2007).

Enquanto transtornos psiquiátricos, a depressão é apontada como o principal fator associado ao comportamento suicida. Considera-se que mais de $50 \%$ dos adolescentes que se suicidaram eram acometidos de depressão maior e, destes, $25 \%$ cometeram alguma tentativa prévia de suicídio, com $15 \%$ de indivíduos consumando o ato (Gómez et al., 2002). No que abrange as variáveis ambientais, as adversidades familiares se apresentam como principal risco de suicídio (Mitchell \& Kim, 2004; Reyes \& Torres, 2001). Entre as variáveis emocionais, aponta-se a repressão emocional, tristeza, necessidade de afeto, busca por valorização, baixa autoestima, sentimentos de culpa e inferioridade, timidez, violência e restrição social como fatores potencialmente de risco a comportamentos suicidas (Campo et al., 2003). 
Na bibliografia disponível também são mencionadas as diferenças de comportamento suicida entre adolescentes com faixa etária maior e menor de 15 anos. Para Bella et al. (2007) e Pfeffer e Shaffer (2001), indivíduos com idade inferior a 15 anos costumam ter seu comportamento suicida atrelado a decisões impulsivas e influências de características familiares, enquanto os casos dos maiores de 15 anos tendem a estar ligados à presença de transtornos mentais e ao consumo de substâncias psicoativas.

A literatura pesquisada repetidamente apresenta que, questões como sentimentos de bem estar, autoestima elevada, capacidade para buscar ajuda em situações necessárias, abertura a novas experiências, flexibilidade emocional e confiança em si mesmo são importantes aspectos que atuam como proteção no que tange ao risco de comportamento suicida. Neste caso, é importante salientar que tais implicações derivam de questões mais amplas como: rede social sólida, relação familiar e integração social (Bastos, 2009; Caycedo et al., 2010; Werlang et al., 2005).

\section{População Adulta}

As taxas mundiais de suicídio tiveram um aumento de $60 \%$ nos últimos 45 anos, sendo que, em alguns países, está entre a principal causa de mortalidade de 15 a 44 anos (Botega et al., 2009; Minayo, Pinto, et al., 2012). No Brasil, as taxas de suicídio em homens com menos de 60 anos atingiu, em 2005, a marca de 11/100mil habitantes, com declínio, em 2006, para 7,1/100mil. Referente ao sexo feminino, a média, em 2006, fora de 3,4 suicídios de homens para cada mulher.

Um estudo realizado em Campinas-SP, por Marín-León e Barros (2003) apontou um aumento nos índices de suicídio de homens e mulheres na fase adulta entre 35-54 anos, no período de 1997-2001, com taxa de 6,6/100mil, indicando assim predominância de óbitos por suicídio na fase adulta. No Rio Grande do Sul, Meneghel et al. (2004) também observaram aumento nas taxas de suicídio, indo de 9/100mil na década de 80 para 11/100mil no final da década de 90 , tendo este nível aumentado mediante a alta taxa de mortalidade masculina.
Estes autores discutem que alguns aspectos do papel social da masculinidade podem ter importante relação nesse sentido, haja vista que questões como reveses econômicos - empobrecimento ou desemprego - são fatores de estresse para o homem que está envolto por uma cultura patriarcal, abalando seu status de provedor. Complementando esta questão, é válido lembrar que a economia brasileira passou por diversas alterações ao longo da década de 90 , período em que o número de suicídios aumentou.

Outra discussão pertinente à população adulta refere-se à questão de que embora ideações suicidas sejam importantes referenciais para possíveis tentativas de suicídio, sua presença isolada não prediz, de maneira segura, futuras tentativas. Logo, é necessário conhecer as demais condutas que apontam para tal viés, tais como indivíduos que se lesionam de maneira consciente, pondo em risco sua vida; transtornos mentais associados com problemáticas psicossociais; bem como antecedentes específicos, indicando assim riscos suicidas (Kapzinski, Quevedo, Schitt, \& Chachamovich, 2001)

Num estudo realizado na Colômbia por Castro-Rueda et al. (2010) com 448 indivíduos e média de idade de 35,9 anos, que realizaram tentativas de suicídio, as estratégias citadas para tal finalidade foram: intoxicação por medicamentos e/ou veneno; autoagressão com arma de fogo ou instrumento perfurante; lançar-se em veículos em movimento; precipitação em locais elevados; bem como outros métodos, podendo levar, quando não consumado, desde danos físicos sem perigo, quanto a danos físicos com risco de vida.

Diversas pesquisas apontam como principais fatores de risco para condutas suicidas: consumo e/ou uso abusivo de álcool e outras substâncias psicoativas; problemas familiares e/ ou parentais; transtornos mentais severos; enfermidades terminais; impulsividade; não possuir parentes e/ou vínculos sociais; rompimento de relações interpessoais significativas; problemas financeiros; histórico familiar de suicídio; abuso na infância, tentativas prévias e ideação suicida; isolamento social; perdas afetivas; histórico familiar de suicídio; transtornos mentais severos, principalmente os transtornos depressivos e do- 
enças terminais; bem como variáveis demográficas e socioeconômicas (Almeida et al., 2009; Meneghel et al., 2004; Rogers, 2001; Weir, 2001; WHO, 2000/2008)

Os principais fatores de risco que implicam condutas suicidas são repetidamente apontados pela literatura como decorrentes de problemas familiares ou sociais, de abuso de substâncias psicoativas, de enfermidades terminais e de transtornos mentais severos. Falando mais especificamente dos problemas familiares ou sociais, comumente tais questões estão atreladas a perdas afetivas, rompimentos interpessoais significativos, a decorrências financeiras e a histórico familiar de suicídio (Almeida et al., 2009; Meneghel et al., 2004; Rogers, 2001; Weir, 2001; WHO, 2000/2008).

No que se refere à hereditariedade do suicídio, Turecki (2001) identificou consistentes evidências, indicando que fatores genéticos possuem importante papel no desempenho do comportamento suicida. Acerca de doenças terminais como fator potencialmente de risco para comportamento suicida, Fanger et al. (2010) discorrem que os fatores associados ao fenômeno em pacientes oncológicos se baseiam nos seguintes pressupostos: sexo masculino, presença de Depressão Maior, declínio das funções físicas, dor, delírio, fadiga, falta de esperança, sensação de desamparo e deficiente apoio social.

Não obstante, levando em consideração que condutas suicidas normalmente ocorrem mediante uma série de fatores correlacionados, a literatura também indica alguns eventos que, quando interligados, potencializam possíveis comportamentos suicidas, tais como: relação entre álcool e transtorno depressivo (King et al., 2006; Lima et al., 2010); transtornos mentais associados (Sher, 2010); perda de vínculos sociais e demais comorbidades (Alves et al., 2004); problemas financeiros e/ou conjugais (Viana et al., 2008), dentre outros.

Outra etapa que merece destaque dentro da população adulta seria o período entre 18 e 24 anos, também comumente chamado de jovem adulto. Para Ores et al. (2012), este período, demarcando a entrada na vida adulta traz consigo transformações onde comportamentos de risco podem se manifestar, inclusive comportamentos suicidas. Em um estudo realizado com 1560 jovens sobre risco de suicídio e comportamentos de risco, com idades entre 18 e 24 anos da cidade de Pelotas, Rio Grande do Sul, apontou que a prevalência do risco de suicídio nesta faixa etária foi de 8\% (Ores et al., 2012), enquanto outra pesquisa com jovens do Ensino Médio sobre o suicídio elencou que, dos participantes, $12,1 \%$ já haviam pensado em suicídio, enquanto $11,5 \%$ já o haviam planejado (Jiang et al., 2010). A presença de transtornos psiquiátricos, dependência química, uso abusivo de álcool, somados a condutas de risco, tais como dirigir embriagado; não usar cinto de segurança ou capacete; ultrapassar sinal vermelho no trânsito; sexo sem proteção e outras práticas que atentem contra a vida se apresentam como fatores potencialmente de risco nesta faixa etária (Ores et al., 2012).

Por possíveis fatores protetivos na idade adulta, Castro-Rueda et al. (2010) elencam alguns contribuintes no intuito de evitar condutas suicidas, tais como: presença de razões para viver; responsabilidade para com a família, mais precisamente com o(s) filho(s), bem como possuir filho(s). Somando-se a eles, boas relações familiares e interpessoais; satisfação nas atividades cotidianas; flexibilidade aos problemas e busca de ajuda em momentos críticos também se apresentam como comportamentos preventivos ao comportamento suicida (Caycedo et al., 2010).

\section{População Idosa}

Assim como nas demais faixas etárias, o suicídio na terceira idade também se constitui como um fenômeno importante a ser investigado, levando em consideração que tal população é a que mais cresce, tanto no Brasil quanto na maior parte do Mundo (Minayo \& Cavalcante, 2010). De acordo com Baptista et al. (2006), as taxas de suicídio em pessoas idosas obtiveram um aumento quando comparadas à população jovem, que realiza mais tentativas de suicídio, uma vez que idosos tendem a comunicar menos suas ideações, bem como são mais letais em seus comportamentos suicidas. 
Em âmbito mundial, um estudo multicêntrico sobre comportamento suicida em idosos, realizado pela WHO/Euro Multicentre Study of Suicidal Behavior (De Leo et al., 2001), em 13 países europeus, apontou como resultados que as taxas médias de suicídio entre indivíduos acima de 65 anos chega a 29,3/100mil e as de tentativas de suicídio a 61,4/100mil. Acerca disso, De Leo et al. (2001) discorrem que, nas sociedades europeias ocidentais, as taxas de tentativas de suicídio são atenuadas entre pessoas idosas, havendo, entretanto, um crescimento nas taxas de suicídios consumados.

No Brasil, considerado um país com taxas baixas de suicídio, as de pessoas acima de 60 anos são o dobro da população em geral, com um aumento exponencial no grupo de idosos do sexo masculino (Lovisi et al., 2009; Minayo, Cavalcante, \& Souza, 2006). Dados epidemiológicos brasileiros registram que, na década de 80 , houve 5.953 óbitos por suicídio em idosos, 8.547 na década de 90 e 7.994 no período entre 2000 a 2006. Os meios mais utilizados para tal finalidade pelos homens são: enforcamento; fumaça/ fogo e disparo de arma de fogo; enquanto que os principais meios de suicídio do sexo feminino prefiguram as seguintes categorias: precipitação de local elevado; enforcamento; ingestão de substâncias; fumaça/fogo/chamas, bem como outros meios não especificados (Minayo, Pinto, et al., 2012).

Faz-se necessário considerar alguns fatores potencialmente de risco para a população idosa, haja vista que tais fenômenos se tornam primordiais na identificação de possíveis riscos. Diversas pesquisas trazem dados sobre fatores de risco característicos à terceira idade, tais como: hábito de se ferir; condutas de risco; tentativas prévias de suicídio e manifestação verbal de possível ideação suicida. No que concerne às condutas de risco, são referidas: descuido com medicação, desânimo para com a vida, visitas constantes ao médico com sintomas não definidos, desinteresse no cuidado pessoal, busca repentina por alguma religião, bem como colocar haveres ou pertences em ordem, sem motivos específicos (Baptista et al., 2006; Beeston, 2006; Holkup, 2003; Minayo \& Cavalcante, 2010).
Ademais, dois tipos de fatores podem ser considerados como riscos para o comportamento suicida: (a) fatores situações e (b) fatores sindrômicos. Pelo primeiro fator, são reconhecidas questões como a aposentadoria, morte de pessoas próximas, diagnóstico de uma enfermidade, isolamento social e perda de função social, enquanto pelo segundo fator, entende-se que sejam quadros de depressão, ansiedade, desenvolvimento de transtornos psicológicos e/ou neurológicos, sentimentos de culpa e dependência, rigidez e impulsividade (Beautrais, 2002; Conwell \& Thompson, 2008).

Convém ressaltar que, em pacientes idosos deprimidos, o risco de suicídio é duas vezes maior do que nos não deprimidos (Pearson \& Brown, 2000). Outra condição importante de se aprofundar diz respeito à questão da aposentadoria que, por sua vez, pode implicar mudança radical no cotidiano da pessoa, gerando desde dificuldades na adaptação às mudanças, até perda do sentido da vida, favorecendo assim a manifestação de comportamentos suicidas (Minayo, Meneghel, et al., 2012).

Com efeito, a OMS (WHO, 2000) considera notável este incremento no número de mortes por suicídio na população idosa, quando comparada há 50 anos. Logo, há a necessidade de desenvolver estratégias de amparo a esta população, tratando questões que lhes são preocupantes, tais como: preparação para a aposentadoria; novas atividades para com os idosos, a fim de que possam desenvolver novas funções que lhes sejam agradáveis; espaços de lazer e convívio social, formando assim redes sociais de suporte; bem como uma preparação adequada das equipes de saúde, onde os profissionais possam dar orientações e suporte para atuarem com os idosos e suas demandas (Beeston, 2006).

\section{Considerações Finais}

Tendo em vista o objetivo desta revisão, tal análise permitiu retificar a proposição do comportamento suicida como multifatorial, uma vez que apresenta diversos fatores associados, apresentados ao longo da discussão. A bibliografia disponível também apontou, tanto para 
generalidades em relação aos fatores associados ao comportamento suicida, quanto a especificidades, de acordo com cada etapa do ciclo vital. Contudo, verifica-se a necessidade de maiores informações sobre o comportamento suicida na fase infantil, bem como maiores estudos nacionais quanto ao comportamento suicida na fase idosa, considerando-se que há poucas pesquisas nacionais sobre o tema, e o índice de suicídio nesta idade vem crescendo no país.

No entanto, enfatiza-se que o fenômeno do suicídio, por ser multicausal, em que vários fatores contribuem para tal, deva ser considerado de modo abrangente e não de maneira unifatorial. Além disso, vale pontuar que, mesmo que o comportamento suicida seja apontado como um ato consciente que visa dar cabo à própria vida, nem sempre este é o propósito subjacente que motiva estes comportamentos.

Em alguns casos, esta autoagressão tem a intenção de obter ganhos secundários, tais como atenção, cuidado ou como forma de castigar alguém emocionalmente, assim como forma de "pedido de socorro". Contudo, reconhecer os sinais de alerta dados pelos indivíduos que planejam cometer tal ação contra si é fundamental para evitar a consumação de tal ato.

Percebe-se, também, a importância da continuidade nas investigações sobre os comportamentos suicidas ao longo do ciclo vital, mediante a ampliação e aprofundamento teórico-prático deste fenômeno. Tal condição reflete na expectativa de que, a partir dos conhecimentos produzidos, medidas preventivas ao suicídio e à promoção da qualidade de vida da população possam ser fortalecidas.

\section{Referências}

Alarcão, M. (2006). (Des)equilibrios familiares Uma visão sistêmica (3. ed.). Coimbra, Portugal: Quarteto.

Almeida, S. A. de, Guedes, P. M. M., Nogueira, J. de A., França, U. de L., \& Silva, A. C. de O. (2009). Investigação de risco para tentativa de suicídio em hospital de João Pessoa- PB. Revista Eletrônica de Enfermagem, 11(2), 383-389.
Alves, H., Kessler, F., \& Ratto, R. L. C. (2004). Co-morbidade: Uso de álcool e outros transtornos psiquiátricos. Revista Brasileira de Psiquiatria, 26(1), 51-53.

Baggio, L., Palazzo, L. S., \& Aerts, D. R. G. de C. (2009). Planejamento suicida entre adolescentes escolares: Prevalência e fatores associados. $\mathrm{Ca}$ derno de Saúde Pública, 25(1), 142-150.

Baptista, M. N. (2004). Suicídio e Depressão - Atualizações. Rio de Janeiro, RJ: Guanabara Koogan.

Baptista, M. N., Morais, P. R., Rodrigues, T. de, \& Silva, J. A. da C. (2006). Correlação entre sintomatologia depressiva e prática de atividades sociais em idosos. Avaliação Psicológica, 5(1), 77-85.

Bastos, R. L. (2009). Suicídios, Psicologia e vínculos: Uma leitura psicossocial. Psicologia USP, 20(1), 67-92.

Beautrais, A. L. (2002). A case-control study of suicide and attempted suicide in older adults. Suicide Life Threat Behavior, 32(1), 1-9.

Beeston, D. (2006). Older people and suicide. Stokeon Trent, UK: Centre for Ageing and Mental Health, Staffordshire University.

Bella, M. E., Fernández, A. R., Acevedo, G., \& Willington, J. M. (2007). Análisis de predictores de riesgo en intentos de suicidio infanto juveniles. Revista de Saúde Pública, 4, 132-137.

Bella, M. E., Fernández, R. A., \& Willington, J. M. (2010). Intento de suicídio em niños e adolescentes: depresión y transtorno de conducta disocial como patologías más frecuentes. Archivos Argentinos de Pediatrìa, 108(2), 124-129.

Botega, N. J., Barros, M. B. A., Oliveira, H. B., Dalgalarrondo, P., \& Marín-León, L. (2005). Suicidal behavior in the community: Prevalence and factors associated with suicidal ideation. Revista Brasileira de Psiquiatria, 27(1), 45-53.

Botega, N. J., Marín-León, L., Oliveira, H. B. de., Barros, M. B. de A., Silva, V. F. da., \& Dalgalarrondo, P. (2009). Prevalências de ideação, plano e tentativa de suicídio: Um inquérito de base populacional em Campinas, São Paulo, Brasil. Caderno de Saúde Pública, 25(12), 2632-2638.

Campo, G., Roa, J., Pérez, A., Salazar, O., Piragauta, C., \& López, L. (2003). Intento de suicidio em menores de 14 años atendidos en el hospital Universitário Del Valle, Cali. Comedica, 34(1), 9-16. 
Carmona, A., Arango, A., Castãno, J., Escobar, J., García, C., \& Godoi, S. (2010). Caracterización del intento de suicidio en una población ingresada a um hospital infantil de Manizades (Caldas, Colômbia), 2000-2008. Archivos de Medicina (Manizales), 10(1), 9-18.

Castro-Rueda, V. A., Rangel Martínez-Villalba, A. M., Camacho, P. A., \& Rueda-Jaimes, G. E. (2010). Factores de riesgo y protectores para intento suicida en adultos colombianos con suicidabilidad. Revista Colombiana de Psiquiatria, 39(4), 705-715.

Caycedo, A., Arenas, M. L., Benítez, M., Cavanzo, P., Leal, G., \& Guzmán, Y. R. (2010). Características psicosociales y familiares relacionadas com intento de suicidio en uma población adolescente em Bogotá- 2009. Persona y Bioética, 14(2), 205-213.

Conwell, Y., \& Thompson, C. (2008). Suicidal behavior in olders. Psichiatric Clinic North American, 3(2), 333-356.

Daolio, E. R., \& Silva, J. V. (2009). Os significados e os motivos do suicídio: As representações sociais de pessoas residentes em Bragança Paulista, SP. Bioethikos, 3(1), 68-76.

De Leo, D., Padoani, W., Scocco, P., Lie, D., Bille-Brahe, U., Arensman, E., ...Faria, S. (2001). Attempted and completed suicide in older subjects: Results from the WHO/EURO Multicentre study of suicidal behaviour. International Journal of Geriatric Psychiatry, 16(3), 300-310.

Evans, E., Hawton, K., \& Deeks, J. (2005). The prevalence of suicidal phenomeno in adolescents: A systematic review of population-based studies. Suicide Life Threat Behavior, 35(3), 239-250.

Fanger, P. C., Azevedo, R. C. S. de, Mauro, M. L. F., Lima, D. D., Gaspar, K. C., Silva, V. F. da, ...Botega, N. J. (2010). Depressão e comportamento suicida em pacientes oncológicos hospitalizados: Prevalência e fatores associados. Revista da Associação Médica Brasileira, 56(2), 173-178.

Gómez, C., Rodríguez, N., Bohórquez, A., Díaz, N., Ospina, M., \& Fernández, C. (2002). Factores asociados al intento de suicidio en la Población Colombiana. Revista Colombiana de Psiquiatría, 31(4), 270-286.

Gutiérrez, M. A., Fernández, S., Jiménez, M., Gutiérrez, A. J., \& Tamayo, O. (2009). Modificación de conocimientos sobre conducta suicida en adolescentes y adultos jóvenes con riesgo. $M E$ DISAN, 13(1).
Holkup, P. (2003). Evidence-based protocol elderly suicide: Secondary prevention. Journal of Gerontology Nurse, 29(6), 6-17.

Jiang, Y., Perry, D. K., \& Hesser, J. E. (2010). Adolescent suicide and health risk behaviors: Rhode Island's 2007 youth risk behavior survey. Journal of Preventive Medicine, 38(5), 551-555.

Joe, D., Romes, D., \& Jamieson, P. (2007). Suicide acceptability in related to suicide planning in U.S. adolescents and Young adults. Suicide Life Threat Behavior, 37(2), 165-178.

Kapzinski, F., Quevedo, J., Schitt, R., \& Chachamovich, E. (2001). Emergências Psiquiátricas. Porto Alegre, RS: Artmed.

King, A. L. S., Nardi, A. E., \& Cruz, M. S. (2006). Risco de suicídio em pacientes alcoolistas com depressão. Jornal Brasileiro de Psiquiatria, 55(1), 70-43.

Lakatos, E. M., \& Marconi, M. A. (2003). Fundamentos de metodologia científica (5. ed.). São Paulo, SP: Atlas.

Larranguibel, M., González, P., Martínez, V., \& Valenzela, R. (2000). Factores de riesgo de la conducta suicida em niños y adolescentes. Revista Chilena de Pediatria, 71(3), 183-191.

Lima, D. D., Azevedo, R. C. S. de, Gaspar, K. C., Silva, V. F. da, Mauro, M. L. F., \& Botega, N. J. (2010). Tentativa de suicídio entre pacientes com uso nocivo de bebidas alcoólicas internados em hospital geral. Jornal Brasileiro de Psiquiatria, 59(3), 167-172.

Lovisi, G. M., Santos, S. A., Legay, L., Abelha, L., \& Valencia, E. (2009). Análise epidemiológica do suicídio no Brasil entre 1960 e 2006 . Revista Brasileira de. Psiquiatria, 31(2), 86-93.

Macente, L. B., \& Zandonade, E. (2010). Avaliação da completude do sistema de informação sobre mortalidade por suicídio na região Sudeste, Brasil, no período de 1996 a 2007. Jornal Brasileiro de Psiquiatria, 59(3), 173-181.

Marín-León, L., \& Barros, B. A. (2003). Mortes por suicídio: Diferenças de gênero e nível socioeconômico. Revista de Saúde Pública, 37(3), 357363.

Meneghel, S. N., Victora, C. G., Faria, N. M. X., Carvalho, L. A., \& Falk, J. W. (2004) Características epidemiológicas do suicídio no Rio Grande do Sul. Revista de Saúde Pública, 38(6), 804810 . 
Minayo, M. C. de S., \& Cavalcante, F. G. (2010). Suicídio entre pessoas idosas: Revisão da literatura. Revista de Saúde Pública, 44(4), 750-757.

Minayo, M. C. de S., Meneghel, S. N., \& Cavalcante, F. G. (2012). Suicídio de homens idosos no Brasil. Ciência \& Saúde Coletiva, 17(10), $2665-$ 2674.

Minayo, M. C. de S., Pinto, L. W., Assis, S. G. de, Cavalcante, F. G., \& Mangas, R. M. do N. (2012). Tendência da mortalidade por suicídio na população brasileira e idosa, 1980-2006. Revista de Saúde Pública, 46(2), 300-309.

Minayo, M. C. S, Cavalcante, F. G., \& Souza, E. R. (2006). Methodological proposal for studying suicide as a complex phenomenon. Caderno de Saúde Pública, 22(8), 1587-1596.

Mitchell, A., \& Kim, Y. (2004). Complicated grief in survivors of suicide. Crisis, 25(1), 12-18.

Ores, L. da C., Quevedo, L. de A., Jansen, K., Carvalho, A. B. de, Cardoso, T. A., Souza, L. D. de M., \& Pinheiro, R. T. (2012). Risco de suicídio e comportamento de risco à saúde em jovens de 18 a 24 anos: Um estudo descritivo. Caderno de Saúde Pública, 28(2), 305-312.

Pavez, P., Santander, N., Carranza, J., \& Vera-Villarroel, P. (2009). Factores de riesgo familiares asociados a la conducta suicida en adolescentes com transtorno depresivo. Revista de Medicina Chilena, 137(2), 226-233.

Pearson, J. L., \& Brown, G. K. (2000). Suicide prevention in late life: Directions of suicide for science and practice. Clinical and Psychological Review, 20(6), 685-705.

Pelkonen, M., \& Marttunen, M. (2003). Child and adolescent suicide. Pediatriatrics Drugs, 5(4), 243-265

Pérez-Olmos, I., Sandoval, E. R., Dussán-Buitrago, M. M., \& Ayala-Aguilera, J. P. (2007). Psychiatric and social characterization of suicide attempts treated at a children's clinic, 2003-2005. Revista de Salud Publica, 9(2), 230-240.

Pfeffer, C. R., \& Shaffer, D. (2001). Parámetros prácticos para la evaluación de niños y adolescentes con comportamiento suicida. Revista de Psiquiatria del Uruguay, 65(2), 146-188.

Psic, S. C. C. B. (2011). Factores de riesgo asociados a conductas suicidas en niños y adolescentes. Archivos de Medicina (Manizales), 11(1), 62-67.
Rábago, H. G., Flores, S. J. E., Ureña, G. M. S., Vargas, J. C. T., \& Gámez, J. G. G. (2010). Factores de riesgo, asociados a intento de suicidio, comparando factores de alta y baja letalidad. Revista de Salud Publica, 12(5), 713-721.

Reyes, W., \& Torres, N. (2001). Intento suicida y funcionamiento familiar. Revista Cubana de Medicina General Integral, 17(5), 452-460.

Rogers, J. R. (2001). Theoretical grouding: The "missing link" in suicide research. Journal Counseling \& Development, 79(1), 16-29.

Roy, A. (2005). Childhood trauma and impulsivity. Possible relevance suicidal behavior. Archives of Suicide Research, 9(2), 147-152.

Sher, L. (2010). Neurobiology of suicidal behavior in post traumatic stress disorder. Expert Review Neurotherapeutics, 10(8), 1233-1235.

Silva, S., \& Maia, A. C. (2010). Experiências adversas na infância e tentativas de suicídio em adultos com obesidade mórbida. Revista de Psiquiatria do Rio Grande do Sul, 32(3), 69-72.

Souza, L. D. de M., Silva, R. A. da, Jansen, K., Khun, R. P., Horta, B. L., \& Pinheiro, R. T. (2010). Suicidal ideation in adolescents aged 11 to 15 years: Prevalence and associated factors. Revista Brasileira de Psiquiatria, 32(1), 37-41.

Souza, V. dos S., Alves, M. da S., Silva, L. A., Lino, D. C. S. F., Nery, A. A., \& Casotti, C. A. (2011). Tentativas de suicídio e mortalidade por suicídio em um município no interior da Bahia. Jornal Brasileiro de Psiquiatria, 60(4), 294-300.

Turecki, G. (2001). Suicidal behavior: Is there a genetic predisposition? Bipolar Disorders, 3(6), 335-349.

Viana, G. N., Zenkner, F. de M., Sakae, T. M., \& Escobar, B. T. (2008). Prevalência de suicídio no Sul do Brasil, 2001-2005. Jornal Brasileiro de Psiquiatria, 57(1), 38-43.

Wagner, I., Ireland, M., \& Resnick, M. (2001). Adolescent suicide attempts: Risk and protectors. Pediatric, 107(3), 485-493.

Wasserman, D., Cheng, Q., \& Jiang, G. X. (2008). Global suicide rates among young peaple aged 15-19. World Psychiatry, 4(2), 114-120.

Weir, E. (2001). Suicide: The hidden epidemic. $\mathrm{Ca}$ nadian Medical Association Journal, 165(5), 634-636. 
Werlang, B. S. G., Borges, V. R., \& Fenterseifer, L. (2005). Fatores de risco ou proteção para a presença de ideação suicida na adolescência. Revista Interamericana de Psicología, 39(2), 259-266.

Werlang, B. S. G., \& Botega, N. J. (Orgs.). (2004). Comportamento suicida. Porto Alegre, RS: ArtMed.

World Health Organization. (2000). Prevenção do suicídio: Um manual para profissionais de saúde em atenção primária. Genebra, Suiça: Autor.
World Health Organization. (2001). Prevencion del suicídio: un instrumento para docentes y demás personal institucional. Geneva, Switzerland: Author.

World Health Organization. (2008). World report on violence and health. Geneva, Switzerland: Author. (Original work published 2000)

Recebido: 05/06/2013

$1^{a}$ revisão: 02/09/2013

Aceite final: 04/09/2013 